\title{
Sustained Very Low Disease Activity and Remission in Psoriatic Arthritis Patients
}

\author{
Ennio Lubrano (D) - Fabio Massimo Perrotta - Silvia Scriffignano • \\ Laura C. Coates · Philip Helliwell
}

Received: July 17, 2019 / Published online: August 19, 2019

(C) The Author(s) 2019

\begin{abstract}
Introduction: Sustained remission should be considered the main therapeutic target in psoriatic arthritis (PsA). Very low disease activity (VLDA) and a DAPSA score $\leq 4$ are the most commonly used criteria. The aim of the present study was to evaluate the rate of sustained remission in a group of PsA patients followed in a real-life setting.

Methods: All PsA patients satisfying CASPAR criteria were followed prospectively every 36 months, in a context of clinical practice by January 2013. Sustained remission was defined when patients achieved a DAPSA score $\leq 4$ and/ or VLDA for at least 12 months. The exclusion criterion was the presence of a condition of
\end{abstract}

Enhanced Digital Features To view enhanced digital features for this article go to: https://doi.org/10.6084/ m9.figshare.9426827.

E. Lubrano $(\bowtie)$. F. M. Perrotta · S. Scriffignano Dipartimento di Medicina e Scienze, della Salute "Vincenzo Tiberio", Università degli Studi del Molise, Campobasso, Italy

e-mail: enniolubrano@hotmail.com

L. C. Coates

Department of Orthopaedics, Rheumatology and Musculoskeletal Sciences, University of Oxford,

Oxford, UK

P. Helliwell

Leeds Institute of Rheumatic and Musculoskeletal

Medicine, University of Leeds, Leeds, UK
VLDA or DAPSA $\leq 4$ at the baseline assessment. Kaplan-Meier survival curve was used to evaluate the survival of patients.

Results: A total of 147 PsA patients were evaluated for the study. Of these, 80 performed at least 12 consecutive months of follow-up. The average duration of follow-up was 24 months (range, 12-60 months). At the last follow-up, 22 patients were on csDMARDs treatment while 58 patients were on bDMARDs. Of the 80 patients, $14(17.5 \%)$ achieved a sustained VLDA while 24 (30\%) achieved sustained remission according to the DAPSA criteria. The mean duration of remission in patients achieving VLDA and DAPSA $\leq 4$ was 17 months for both criteria. High baseline levels of CRP, shorter disease duration, and less pain at baseline were found to be predictors of sustained VLDA and DAPSA remission.

Conclusions: In our study, based on clinical practice, a sustained VLDA was achieved in $17.5 \%$ and a sustained remission according to the DAPSA criteria in 30\% of patients with PsA.

Keywords: Psoriatic arthritis; Remission; Very low disease activity

\section{INTRODUCTION}

Psoriatic arthritis (PsA) is a chronic inflammatory disease with a prevalence of about $13.8-30 \%$ among patients with psoriasis and 
with an incidence of 22.59 cases/100,000/year [1]. Peripheral joint involvement and the presence of enthesitis lead to cartilage and bone damage with functional impairment and reduced quality of life [2]. Furthermore, the possible presence of severe skin involvement, extra-articular manifestations, and comorbidities reinforces the need for the use of effective drugs to control disease activity and improve the overall outcome [3]. In PsA patients, a state of remission or low disease activity was identified as the target of therapy, and recently different criteria were proposed and validated. The Disease Activity index for PSoriatic Arthritis (DAPSA) [4] and the Minimal Disease Activity (MDA) criteria [5] were included in the recent treat-to-target recommendations [6]: the DAPSA is considered as a unidimensional index focused on articular involvement while the MDA explores different disease domains including function. The importance of disease control with the lowest grade of disease activity is justified by the fact that achieving sustained MDA (defined as MDA for over 12 months at consecutive clinic visits) reduces radiographic joint damage progression over a 3-year period [7]. In a more recent study, the same authors proposed a more stringent definition of remission (very low disease activity, VLDA) in which all $7 / 7$ criteria previously defined had to be satisfied [8]. Although different observational studies showed the efficacy and effectiveness of different treatment strategies in the induction of a status of MDA and remission, only a few reports are available on the rate of PsA patients achieving sustained remission, using these more stringent criteria, in a real-life setting.

In this scenario, the aim of this study was to identify sustained VLDA and sustained remission (DAPSA score $\leq 4$ ) in a retrospective group of PsA patients treated in a real-life setting. A secondary aim was to evaluate predictive factors for sustained VLDA or remission.

\section{METHODS}

In this retrospective analysis of longitudinal cohort, patients were selected from the database of the Rheumatology Unit, Department of
Medicine and Health Science-University of Molise. All patients fulfilled the ClASsification criteria for Psoriatic ARthritis (CASPAR) criteria [9].

The study protocol was in compliance with the Declaration of Helsinki and written consent was obtained from each participant. The study was approved by the Institutional Review Board of the University of Molise (protocol n. 0001-09-2017).

\section{Data Collection}

All PsA patients in our database from January 2013 were potentially considered eligible for the study.

Exclusion criteria were as follows:

1. Patient's age $<18$ years.

2. A baseline DAPSA $\leq 4$ or a condition of VLDA at first baseline visit.

3. Patients with $<12$ months follow-up.

Patient data collection included a medical history, physical examination, current use of medications, and laboratory assessment. Demographics and disease characteristics including age, gender, disease duration, and pattern of articular manifestation were taken into account. The clinical assessment encompassed the number of tender joints (of the 68 assessed joints) and swollen joints (total of 66 joints), enthesitis, and dactylitis. Enthesitis was measured using the Leeds Enthesitis Index (LEI) [10], and dactylitis as present/absent. Skin assessment included the Psoriasis Area Severity Index (PASI) score and the body surface area (BSA) [11]. The Health Assessment Questionnaire (HAQ) [12] was used to assess function. Patient Global Assessment (PtGA) and pain assessment on visual analogue scale (VAS) were performed by all patients. Physician's global evaluation of disease activity on a VAS scale was also recorded [13]. C reactive protein (CRP) was also collected.

Only patients who completed the required follow-up period were extracted from the database. VLDA was identified according to Coates et al. and was satisfied when all seven criteria were met [8]. DAPSA score was identified 
according to Nell-Duxneur et al. and was calculated by adding the number of tender and swollen joints, VAS pain, PtGA, and CRP (mg/ dl). DAPSA score $\leq 4$ identified remission [4]. Sustained VLDA and sustained DAPSA remission was defined as the presence of these conditions for over 12 months at consecutive clinic visits [7].

\section{Statistical Analysis}

Categorical variables were analyzed by $\chi^{2}$ test with Yates' correction or Fisher's exact test. Percentages were used when necessary. The significance of the differences was determined using the Mann-Whitney test for unpaired samples and Wilcoxon's test for paired samples. Kaplan-Meier (KM) survival curves were plotted to determine the rates of VLDA and DAPSA remission. In KM survival curves calculation, we entered time until the subject was "censored" or the "event" occurred. The probability of achieving sustained VLDA and/or DAPSA remission was explored using an odds ratio (OR-lower and upper 95\% CI) of outcome relative to the main variables.

The results were expressed as median (25th-75th percentile) for non-normally distributed data or mean (SD) for normally distributed data. $P$ values $<0.05$ were considered significant.

\section{RESULTS}

Of the 147 PsA patients present in our database, 80 had a follow-up of $>12$ months and were considered eligible for the analysis. Table 1 shows the baseline patients' clinical characteristics.

The mean duration of follow-up in our group was 24 months (range, 12-60 months). Of the 80 patients selected, at the last follow-up, 22 were on csDMARDs treatment while 58 were on bDMARDs (40 in bDMARDs monotherapy and 18 in association with csDMARDs). Of these patients, during the follow-up, 20 (25\%) and 28 (35\%) achieved a condition of VLDA and DAPSA remission for at least one time, while 14 (17.5\%) achieved a sustained VLDA and 24
Table 1 The main demographic and clinical features of PsA patients $(n=80)$, at baseline

\begin{tabular}{ll}
\hline Male/female & $49 / 31$ \\
Age, years (SD) & $55(16.07)$ \\
Disease duration, years (median/25th-75th percentile) & $5(2-9)$ \\
Articular manifestations (\%) & \\
Axial & 10.8 \\
Peripheral arthritis & 81.6 \\
Enthesitis & 24.4 \\
Dactylitis & 20.4 \\
Extra-articular manifestations (\%) & \\
Uveitis & 4 \\
Psoriasis & \\
IBD & 95.2 \\
BMI (mean/SD) & 2.7 \\
DAPSA (mean/SD) & $27.3 / 4.5$ \\
CRP, mg/dl (mean/SD) & $14.6 / 10.3$ \\
CRP < 5 mg/l, $n$ (\%) & $0.63 / 0.95$ \\
PtGA (mean/SD) & $35(44.8)$ \\
VAS pain (mean/SD) & $52.2 / 24$ \\
VAS physician (mean/SD) & $52 / 25.7$ \\
HAQ (mean/SD) & $44.1 / 23.5$ \\
PASI (median/25th-75th percentile) & $0.70 / 0.63$ \\
PASI $\leq 3$ (\%) & $1.6 / 0.9-7.4$ \\
PASI $>3$ and $\leq 10(\%)$ & 70 \\
PASI $>10(\%)$ & 17.5 \\
LEI (mean/SD) & 12.5 \\
Treatment & $0 / 0-1$ \\
csDMARDs, $n(\%)$ & \\
Anti-TNF, $n(\%)$ & $26(32.5)$ \\
Anti_IL-12/23, $n(\%)$ & $30(37.5)$ \\
Anti-IL-17, $n(\%)$ & $3.7)$ \\
Apremilast & \\
\hline & \\
&
\end{tabular}

(30\%) a sustained DAPSA remission (Fig. 1). The mean duration of remission in patients achieving VLDA and DAPSA $\leq 4$ was 17 months for both criteria. Figure 2 shows the Kaplan-Meier curve for VLDA and DAPSA remission during the follow-up.

In our group of patients, male sex (only for DAPSA remission), shorter disease duration, less pain at baseline and the presence of higher baseline values of CRP were found to be predictors of sustained VLDA and DAPSA remission (Table 2). 


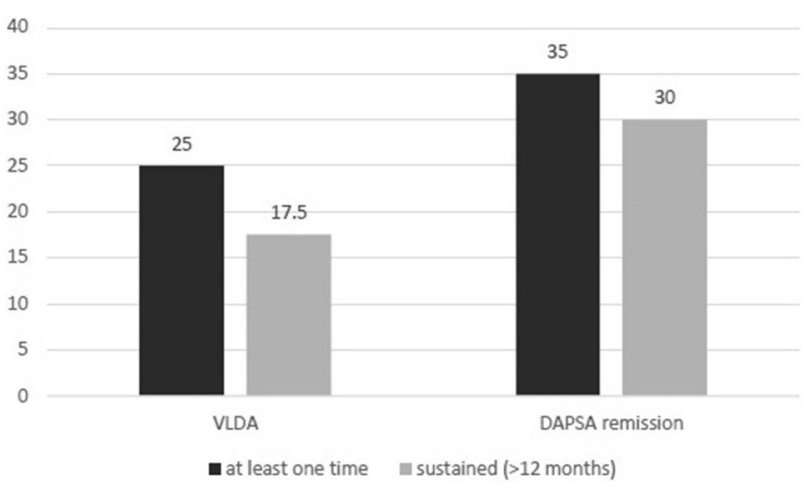

Fig. 1 Rate of PsA patients $(n=80)$ achieving VLDA and DAPSA remission for at least one time and for a sustained (>12 months) period
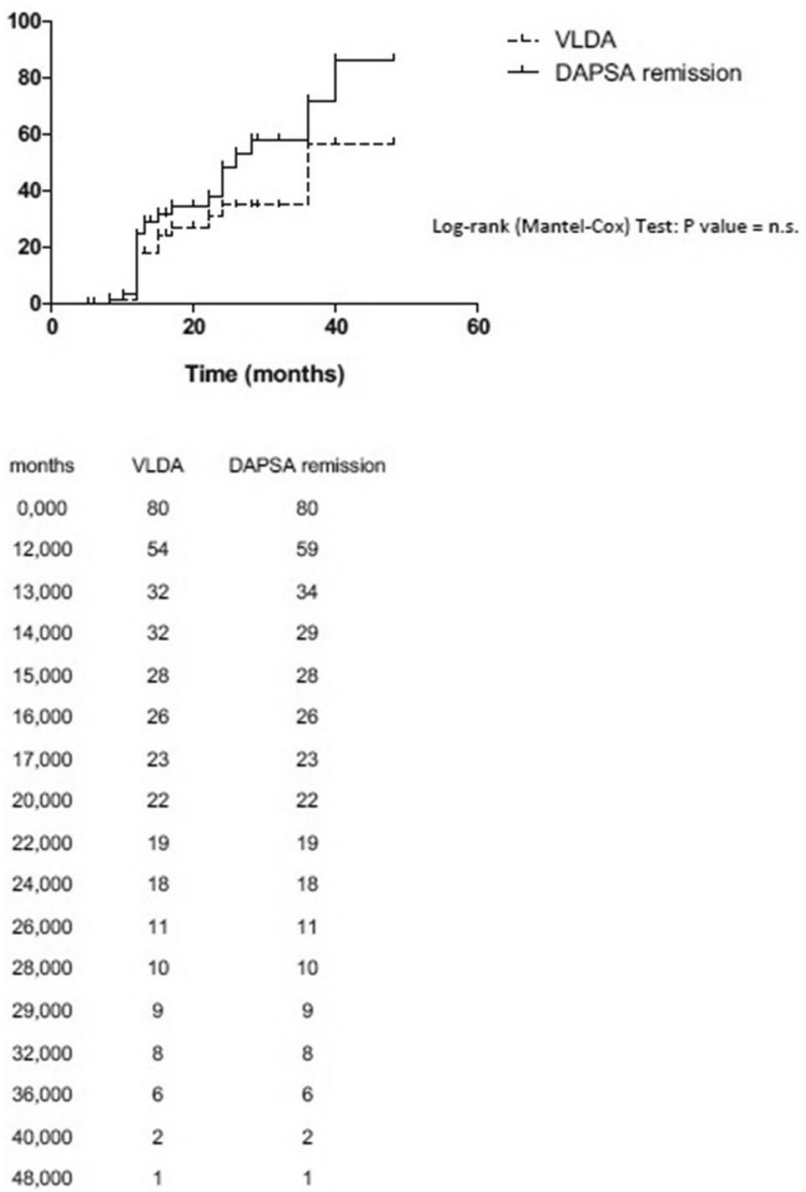

Fig. 2 Kaplan-Meier survival curves for VLDA and DAPSA remission during the follow-up in our group of PsA patients 
Table 2 ORs (95\% CI) of achieving sustained VLDA and DAPSA remission according to the baseline variable values

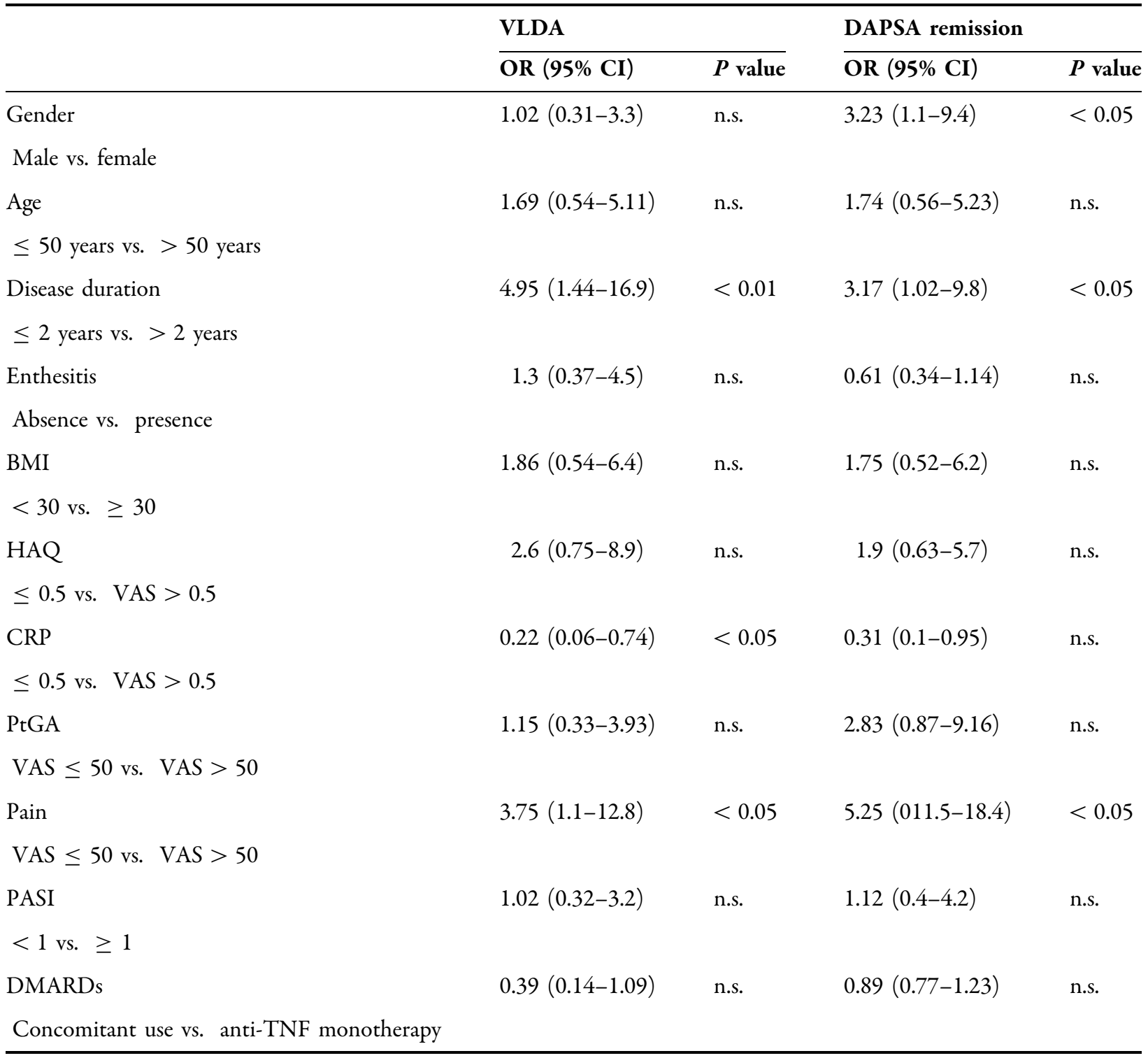

\section{DISCUSSION}

Remission or low disease activity is the treatment goal in chronic inflammatory arthritis and sustained low disease activity proved to be effective in reducing radiographic progression of PsA. Previous studies reported that a state of "remission" (defined using non-validated criteria) could be achieved by $17.6-25 \%$ of PsA patients treated with synthetic DMARDs $[14,15]$ and, about $30-60 \%$ of patients treated with biologic drugs in both clinical trials and observational studies can achieve a condition of MDA [16-21]. However, stringent definitions of remission such as VLDA and DAPSA are more difficult to reach. Moreover, residual disease activity can be found in patients achieving low disease activity or MDA [22, 23] suggesting that stringent definitions of remission are required to obtain comprehensive disease control. Finally, due to fluctuation of disease activity, remission may be lost during follow-up. Thus, 
sustained remission could represent a more reliable index of optimal disease control and even a predictor of no progression of the disease [7]. In our study, 25-35\% of PsA patients achieved VLDA and DAPSA remission for at least one time during the follow-up. Furthermore, 17.5 and $30 \%$ reached a sustained remission. Given these data, sustained VLDA seems to be more difficult to achieve, since, as a multi-dimensional index, it comprises domains other than joints (skin, enthesis, function).

In our study, our results showed that the probability to obtain a status of sustained VLDA or DAPSA remission is linked to a shorter disease duration, high levels of baseline CRP, and less pain. This is in keeping with previous studies reporting these factors as good predictors of outcome [16, 24].

Finally, the study had some limitations: first, the retrospective design that could allow some bias and second, the relatively small number of patients with a long follow-up. Nevertheless, the study may contribute to better understanding this intriguing topic.

\section{CONCLUSIONS}

In conclusion, despite the relatively small number of patients, the present study showed that sustained VLDA or DAPSA remission status might be achieved by PsA patients.

\section{ACKNOWLEDGEMENTS}

We thank the participants of the study.

Funding. No funding or sponsorship was received for this study or publication of this article.

Authorship. All named authors meet the International Committee of Medical Journal Editors (ICMJE) criteria for authorship for this article, take responsibility for the integrity of the work as a whole, and have given their approval for this version to be published.
Authorship Contributions. All authors have made substantial contributions to all of these sections: conception and design of the study, acquisition of data, analysis and interpretation of data, drafting the article, revising it critically for important intellectual content, and final approval of the version to be submitted.

Disclosures. Ennio Lubrano is a member of the journal's Editorial Board. Laura C. Coates is a member of the journal's Editorial Board. Philip Helliwell is a member of the journal's Editorial Board. Fabio Massimo Perrotta and Silvia Scriffignano have nothing to disclose.

Compliance with Ethics Guidelines. The study protocol was in compliance with the Declaration of Helsinki and written consent was obtained from each participant. The study was approved by the Institutional Review Board of the University of Molise (protocol n. 0001-092017).

Data Availability. The datasets generated during and/or analyzed during the current study are not publicly available but are available from the corresponding author upon reasonable request.

Open Access. This article is distributed under the terms of the Creative Commons Attribution-NonCommercial 4.0 International License (http://creativecommons.org/licenses/ by-nc/4.0/), which permits any noncommercial use, distribution, and reproduction in any medium, provided you give appropriate credit to the original author(s) and the source, provide a link to the Creative Commons license, and indicate if changes were made.

\section{REFERENCES}

1. De Socio A, Perrotta FM, Grasso GM, Lubrano E. Incidence of rheumatoid arthritis, psoriatic arthritis and polymyalgia rheumatica in an inland area of central Italy: results of the CAMPO-RHE study. Postgrad Med. 2018;130(1):137-41. 
2. Ritchlin CT, Colbert RA, Gladman DD. Psoriatic arthritis. N Engl J Med. 2017;376:957-70. https:// doi.org/10.1056/NEJMra1505557.

3. Helliwell P, Coates L, Chandran V, Gladman D, de Wit M, FitzGerald $\mathrm{O}$, et al. Qualifying unmet needs and improving standards of care in psoriatic arthritis. Arthritis Care Res (Hoboken). 2014;66:1759-66.

4. Schoels MM, Aletaha D, Alasti F, Smolen JS. Disease activity in psoriatic arthritis (PsA): defining remission and treatment success using the DAPSA score. Ann Rheum Dis. 2016;75:811-8.

5. Coates LC, Fransen J, Helliwel PS. Defining disease activity in psoriatic arthritis: a proposed objective target for treatment. Ann Rheum Dis. 2010;69:48-53.

6. Smolen JS, Schöls M, Braun J, Dougados M, FitzGerald O, Gladman DD, et al. Treating axial spondyloarthritis and peripheral spondyloarthritis, especially psoriatic arthritis, to target: 2017 update of recommendations by an international task force. Ann Rheum Dis. 2018;77:3-17.

7. Coates LC, Cook R, Lee KA, Chandran V, Gladman DD. Frequency, predictors, and prognosis of sustained minimal disease activity in an observational psoriatic arthritis cohort. Arthritis Care Res (Hoboken). 2010;62:970-6.

8. Coates LC, Helliwell PS. Defining low disease activity states in psoriatic arthritis using novel composite disease instruments. J Rheumatol. 2016;43:371-5.

9. Taylor W, Gladman D, Helliwell P, Marchesoni A, Mease P, Mielants H, CASPAR Study Group. Classification criteria for psoriatic arthritis: development of new criteria from a large international study. Arthritis Rheum. 2006;54:2665-73.

10. Healy PJ, Helliwell PS. Measuring clinical enthesitis in psoriatic arthritis: assessment of existing measures and development of an instrument specific to psoriatic arthritis. Arthritis Rheum. 2008;59:686-91.

11. Fredriksson T, Pettersson U. Severe psoriasis-oral therapy with a new retinoid. Dermatologica. 1978;157:238-44.

12. Ranza R, Marchesoni A, Calori G, Bianchi G, Braga $M$, Canazza $S$, et al. The Italian version of the Functional Disability Index of the Health Assessment Questionnaire. A reliable instrument for multicenter studies on rheumatoid arthritis. Clin Exp Rheumatol. 1993;11:123-8.
13. Lubrano E, Perrotta FM, Parsons WJ, Marchesoni A. Patient's global assessment as an outcome measure for psoriatic arthritis in clinical practice: a surrogate for measuring low disease activity? J Rheumatol. 2015;42:2332-8.

14. Gladman DD, Hing EN, Schentag CT, Cook RJ. Remission in psoriatic arthritis. J Rheumatol. 2001;28:1045-8.

15. Lubrano E, Perrotta FM, Kavanaugh A. An overview of low disease activity and remission in psoriatic arthritis. Clin Exp Rheumatol. 2015;33(5 Suppl 93):S51-4.

16. Perrotta FM, Marchesoni A, Lubrano E. Minimal disease activity and remission in psoriatic arthritis patients treated with anti-TNF- $\alpha$ drugs. J Rheumatol. 2016;43:350-5.

17. Lubrano E, Parsons WJ, Perrotta FM. Assessment of response to treatment, remission, and minimal disease activity in axial psoriatic arthritis treated with tumor necrosis factor inhibitors. J Rheumatol. 2016;43:918-23.

18. Perrotta FM, Lubrano E. Subcutaneous anti-TNF alfa induced sustained minimal disease activity and remission in psoriatic arthritis patients: a retrospective study. Postgrad Med. 2016;128:693-6.

19. Lubrano E, Mesina F, Caporali R. Clinical remission in rheumatoid arthritis and psoriatic arthritis. Clin Exp Rheumatol. 2018;36(5):900-10.

20. Coates LC, Rahman P, Psaradellis E, Rampakakis E, Osborne B, Lehman AJ, Nantel F. Validation of new potential targets for remission and low disease activity in psoriatic arthritis in patients treated with golimumab. Rheumatology (Oxford). 2019;58(3):522-6.

21. Coates LC, Mease PJ, Gossec L, Kirkham B, Sherif B, Gaillez $\mathrm{C}$, et al. Minimal disease activity among active psoriatic arthritis patients treated with secukinumab: 2-year results from a multicenter, randomized, double-blind, parallel-group, placebocontrolled phase III study. Arthritis Care Res (Hoboken). 2018;70:1529-35.

22. Coates LC, Lubrano E, Perrotta FM, Emery P, Conaghan PG, Helliwell PS. What should be the primary target of "treat to target" in psoriatic arthritis? J Rheumatol. 2019;46:38-42.

23. Lubrano E, De Socio A, Perrotta FM. Comparison of composite indices tailored for psoriatic arthritis treated with csDMARD and bDMARD: a cross-sectional analysis of a longitudinal cohort. J Rheumatol. 2017;44:1159-64. 
24. Theander E, Husmark T, Alenius GM, Larsson PT, Teleman A, Geijer M, et al. Early psoriatic arthritis: short symptom duration, male gender and preserved physical functioning at presentation predict favourable outcome at 5-year follow-up. Results from the Swedish Early Psoriatic Arthritis Register (SwePsA). Ann Rheum Dis. 2014;73:407-13. 